\title{
En focus in HCB
}

\author{
Douglas J. Taatjes ${ }^{1}$ Jürgen Roth ${ }^{2}$
}

Published online: 4 February 2022

(c) The Author(s), under exclusive licence to Springer-Verlag GmbH Germany, part of Springer Nature 2022

In this second Editorial for 2022, we will highlight three Original Articles describing (1) the effect of melanoma-produced exosomes on melanoma-associated fibroblasts compared to normal dermal fibroblasts, (2) exploitation of expression of altered focal adhesion kinase protein and mRNA for analysis of papillary thyroid carcinoma progression, and (3) detemination of the bioavailability time of thymidine analogues in an in vivo mouse model; and a Short Communication describing multimodal chemical analysis and machine learning to assess the effects of environmental pollutants on a soil earthworm model. We hope you enjoy these highlights as well as the entire February issue of Histochemistry and Cell Biology.

\section{Melanoma cell-derived exosomes and communication in the melanoma microenvironment}

Exosomes are extracellular vesicles 40-100 nm in diameter formed by the secretion of multivesicular endosomes of many different cell types (Harding et al. 1983; Pan and Johnstone 1983; Raposo and Stoorvogel 2013). They play important roles in intercellular communication as vehicles for the transfer of membrane and cytosolic proteins, lipids and RNA (both microRNA and mRNA). Cancer cells and other cell types in the tumor microenvironment secrete exosomes, which may contribute to tumor progression and tumor cell migration related to metastasis formation (Rak 2010; Hood et al., 2011). Regarding exosomes secreted by melanoma cells, they may stimulate proinflammatory signaling in the metastatic microenvironment of melanoma (Gener Lahav et al. 2019), suppress antitumoral activity of $\mathrm{CD}^{+}$T lymphocytes (La Shu et al. 2020), and be

Douglas J. Taatjes

douglas.taatjes@med.uvm.edu

1 Department of Pathology and Laboratory Medicine, Larner College of Medicine, University of Vermont, Burlington, VT 05405, USA

2 University of Zurich, 8091 Zurich, Switzerland involved in the reprogramming of fibroblasts to cancer-associated fibroblasts (Hu and $\mathrm{Hu} 2019$; La Shu et al. 2018).

In their present work, Strnadová et al. (2022) investigated the effect of melanoma cell-derived exosomes on cultured normal human dermal fibroblasts (HDF) and on melanomaassociated fibroblasts (mCAF) alone and in combination with melanoma cells. Among three melanoma cell lines (A2058, BLM, and G361) analyzed, the G361 cell line, established from a primary malignant melanoma, provided a rich source of exosomes. Exosomes purified from G361 cells significantly decreased adhesion and growth of both HDF and mCAF, with the effect being stronger on HDF, as well as their proliferation, and increased the speed of migration in 2D G361 exosome-exposed HDF but not of mCAF. For both types of fibroblasts, when grown as 3D spheroids in agarose molds, treatment with G361-derived exosomes significantly increased their mean invasion index after 24 and 48 h of treatment. Mixed 3D spheroids composed of G361 melanoma cells and either fibroblast type were exposed to a mixture of collagen-1 and G361-derived exosomes, and the melanoma cell invasion was evaluated after $144 \mathrm{~h}$. The invasion of melanoma cells of spheroids formed with HDF was not significantly influenced by G361 exosomes, whereas their invasion was significantly increased when spheroids were formed with mCAF (Fig. 1).

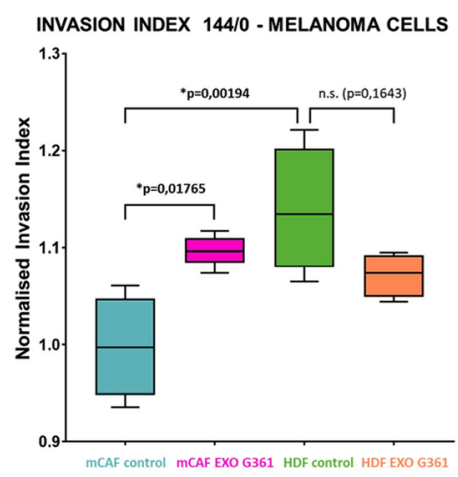

Fig. 1 Normalized invasion index of melanoma cells grown as spheroids with either melanoma-associated fibroblasts (mCAF) or normal human dermal fibroblasts (HDF). From Strnadová et al. (2022) 
In their transcriptomic analysis, the authors detected a significant, albeit different influence of G361 exosomes on fibroblasts. For instance, although genes for cytokines and chemokines were upregulated in mCAF, they were unchanged or downregulated in HDF. Genes for various matrix metalloproteinases were generally upregulated in mCAF and only infrequently in HDF. Gene set enrichment analysis verified that G361 exosomes had a major influence on proteins involved in extracellular matrix organization and inflammatory response in both HDF and mCAF. Analysis of supernatants from control and G361 exosome-treated HDF and mCAF cultures by a Proteome Profiler XL kit revealed a complex pattern. For example, IL1RL1 was detected in non-treated HDF and in G361 exosome-treated mCAF, whereas thrombospondin-1 was found in G361 exosome-treated HDF and in non-treated mCAF. On the other hand, IL-6 and CXCL-8 were significantly increased in exosome-treated mCAF but remained unchanged in HDF. In more general terms, the authors concluded that activation of mCAF by G361derived exosomes occurs more commonly than that of HDF. As a consequence of the observed significantly stimulated tumor-promoting proinflammatory activity of G361 exosomes on mCAF, it is proposed that they may represent a therapeutic target.

\section{Expression of focal adhesion kinase to monitor papillary thyroid carcinoma progression}

Focal adhesion kinase (FAK), a non-receptor tyrosine kinase localized to the cell cytoplasm, plasma membrane, and nucleus, is overexpressed in a number of human cancers and is involved in several aspects of tumor progression (Zhou et al. 2019). Ignjatovic et al. (2022) have now investigated whether expression of altered and unaltered FAK at the protein and mRNA level may serve as molecular markers for diagnosing progression of papillary thyroid carcinoma (PTC), a normally non-aggressive tumor which in some cases can progress to metastatic spread. Specifically, they analyzed expression of autophosphorylated FAK on tyrosine residue in position 397 of the protein (pY397-FAK; indicates FAK protein in activated state) as well as two aberrantly spliced FAK transcripts (FAK-Del126 and FAK-Del133) since all three have been previously shown to be present in some other human cancers (Albasri et al. 2014; Fang et al. 2014a, b; Yao et al. 2014). Employing Western blotting and immunohistochemistry (Fig. 2) to detect FAK protein and qRT-PCR for mRNA transcripts, they analyzed samples in a semi-quantitative manner

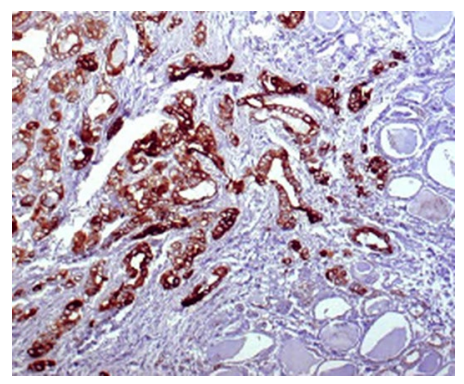

Fig. 2 Autophosphorylated focal adhesion kinase (pY397-FAK) is detectable by immunohistochemistry in papillary thyroid carcinoma but not in surrounding normal tissue. From Ignjatović et al. (2022)

from 80 patients diagnosed with PTC (both tumor and paired non-malignant samples were compared).

Their results demonstrated (1) the expression of pY397-FAK was found to be elevated in the malignant samples compared to the non-malignant controls by both Western blot and immunohistochemical analyses; (2) pY397-FAK was found to be predominantly distributed in both the cell cytoplasm and plasma membrane, with less frequent occurrence in the nucleus; (3) when present, nuclear localization of pY397-FAK was most commonly associated with a follicular variant of PTC; (4) expression of spliced FAK-Del126 and FAK-Del133 transcripts was found to correlate well with the expression of pY397-FAK protein in tumor samples; (5) both pY397-FAK protein expression and FAK-Del133 mRNA expression correlated positively with the degree of tumor neoplastic tissue infiltration. Their results indicate that the level of PTC aggressiveness can be correlated with a deregulation of FAK at both the protein and mRNA levels. Moreover, the expression of pY397-FAK protein may prove to be a potential biomarker for evaluating the aggressive nature of individual PTC tumors.

\section{In vivo bioavailability estimates for thymidine analogues}

Cellular DNA synthesis and cell proliferation are often investigated using the incorporation of labeled thymidine (or its synthetic analogues) into replicating DNA (Manders et al. 1996). When seeking to investigate cell cycles in cultured cells, modified nucleotides are typically added to the cell culture medium, followed by analysis of their incorporation into the DNA. Similar efforts in intact animals are more complicated, where the time interval during which the labeled nucleotide incorporates into the replicating DNA is difficult to control via the methodology applied; instead, in this case, 
the time interval can be estimated by the rate of label propagation in the blood system together with the rate of the metabolism of the label in the organism. This raises the important concept of bioavailability time, which is defined as "the time interval in which the entire amount of the modified nucleotide is completely metabolized" (Maltsev et al. 2022). Since Podgorny et al. (2018) have recently published a triple $\mathrm{S}$-phase labeling of dividing stem cells in vivo using combinations of the thymidine analogues 5-iodo-2'-deoxyuridine (IdU), 5-chloro-2'deoxyuridine (CldU), and 5-ethynyl-2'-deoxyuridine (EdU), they have now investigated the bioavailability time for each of these modified nucleotides (Maltsev et al. 2022) to determine the level of consistency among these labeling probes. They evaluated the labeling kinetics in vivo and the time for clearance from the blood for the common thymidine analogues IdU, CldU, and EdU and compared them to those for the standard BrdU. A two-pronged approach for evaluation was utilized: (1) using an in vivo mouse model they determined the number of labeled cells in the hippocampal dentate gyrus (DG) at different time points following injection of the synthetic probes (the DG possesses many positive attributes which make it a good model tissue area for assessing cell cycle labeling) (Fig. 3); (2) blood sera collected from the same injected animals was incubated with cultured HeLa cell media to investigate the ability of cleared nucleotides to label DNA synthesis in vitro.

Their results demonstrated that all of the thymidine analogues show similar cell labeling kinetics (vast majority of dividing cells labeled within first $10 \mathrm{~min}$ of pulse label delivery) and are also cleared from the circulation with very comparable time intervals following a single intraperitoneal injection. Specifically, times of label incorporation were determined to be on the order of $1 \mathrm{~h}$. As such, sera collected from the animals within $1 \mathrm{~h}$ of thymidine analogue injection contained sufficient amounts of these probes to label proliferating cells in culture. These results validate the use of multiple

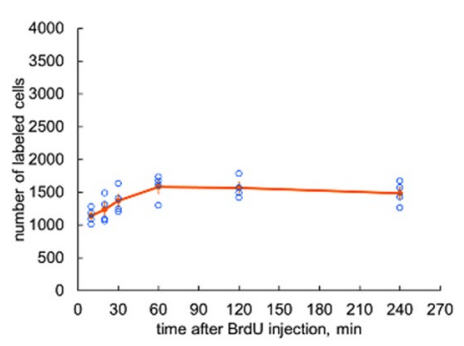

Fig. 3 Labeling kinetics of hippocampal dentate gyrus cells after intraperitoneal injection of the thymidine analogue BrdU. From Maltsev et al. (2022) popular thymidine analogues, either singly or in multiple S-phase labeling studies, provided that they are used in equimolar concentrations.

\section{Sequential multimodal chemical image analysis and machine learning for environmental toxicology}

Knowledge about the uptake of environmental pollutants and their effects on tissue integrity and function is of ever increasing importance (Leblanc 2004). The earthworm Eisenia fetida is a terrestrial model organism for toxicological studies, and in addition to histologicalhistochemical techniques, chemical imaging analyses by different spectroscopic techniques have been applied (Liebeke et al. 2015). However, traditional tissue analysis approaches are not only time-consuming but also of limited value. In their present work, Ritschar et al. (2022) aimed to improve this situation by developing a multimodal approach and combining it with improved data acquisition and evaluation. They report a sequential multimodal imaging approach combining Fourier transform infrared spectroscopy (FTIR) followed by matrixassisted laser desorption/ionization mass spectrometry imaging (MALDI-MSI) and finally H\&E staining on the same $20-\mu \mathrm{m}$-thick cryosections prepared from sodiumcarboxymethylcellulose-embedded segments of adult Eisenia fetida (Fig. 4).

By first applying FTIR imaging, a rapid, noninvasive chemical overview of the tissue section was obtained and used to identify regions of interest for subsequent MS imaging. Analysis of the FTIR spectra was performed by applying a machine learning algorithm based on random decision forest classification (RDF) (Horning 2010), which is highly capable of dealing with complex data systems. The authors emphasized several advantages of RDF, such as rapid tissue type characterization and reduced observer bias providing a high percentage of classification accuracy. Following FTIR identification of regions of interest, specific lipids were identified by high-resolution MALDI-MSI. MALDI-MSI, as a label-free on-tissue type of biochemical lipid microscopy

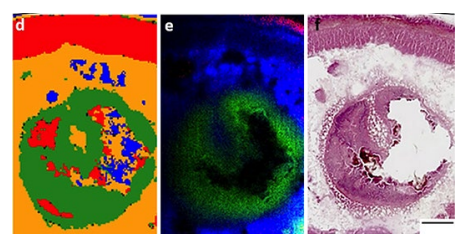

Fig. 4 Region of interest of a tissue section of E. fetida. d Results of RDF model application of the FTIR data. e Different lipids identified by MALDI-MSI are colored in red, blue, and green. f H\&E staining, bright-field microscopy. From Ritschar et al. (2022) 
(Sparvero et al. 2012), is particularly suitable for this analysis since lipids are the main tissue constituents of Eisenia fetida and their metabolism has been shown to be altered following pollutant exposure (Mayilswami et al. 2017). The optional H\&E staining as a last step provides correlation of the FTIR and MALDI-MSI data with histological features. Correctly, the authors conclude that their fast and non-invasive multimodal workflow has great potential for the analysis of model organisms in ecotoxicological research.

\section{References}

Albasri A, Fadhil W, Scholefield JH, Durrant LD, Ilyas M (2014) Nuclear expression of phosphorylated focal adhesion kinase is associated with poor prognosis in human colorectal cancer. Anticancer Res 34:3969-3974

Fang XQ, Liu XF, Yao L et al (2014a) Somatic mutational analysis of FAK in breast cancer: a novel gain-of-function mutation due to deletion of exon 33. Biochem Biophys Res Commun 443:363369. https://doi.org/10.1016/j.bbrc.2013.11.134

Fang XQ, Liu X, Yao L et al (2014b) New insights into FAK phosphorylation based on a FAT domain-defective mutation. PLoS ONE 9:e107134. https://doi.org/10.1371/journal.pone.0107134

Gener Lahav T, Adler O, Zait Y et al (2019) Melanoma-derived extracellular vesicles instigate proinflammatory signaling in the metastatic microenvironment. Int J Cancer 145:2521-2534. https://doi. org/10.1002/ijc.32521

Harding C, Heuser J, Stahl P (1983) Receptor-mediated endocytosis of transferrin and recycling of the transferrin receptor in rat reticulocytes. J Cell Biol 97:329-339. https://doi.org/10.1083/jcb.97.2. 329

Hood JL, San RS, Wickline SA (2011) Exosomes released by melanoma cells prepare sentinel lymph nodes for tumor metastasis. Cancer Res 71:3792-3801. https://doi.org/10.1158/0008-5472. CAN-10-4455

Horning N (2010) Random forests: an algorithm for image classification and generation of continuous fields data sets. Int Conf Geoinform Spat Infrastruct Dev Earth Allied Sci 2010:1-6

Hu T, Hu J (2019) Melanoma-derived exosomes induce reprogramming fibroblasts into cancer-associated fibroblasts via Gm26809 delivery. Cell Cycle 18:3085-3094. https://doi.org/10.1080/ 15384101.2019.1669380

Ignjatovic VB, Jankovic Miljus JR, Roncevic JV et al (2022) Focal adhesion kinase splicing and protein activation in papillary thyroid carcinoma progression. Histochem Cell Biol. https://doi. org/10.1007/s00418-021-02056-y

La Shu S, Yang Y, Allen CL et al (2018) Metabolic reprogramming of stromal fibroblasts by melanoma exosome microRNA favours a pre-metastatic microenvironment. Sci Rep 8:12905. https:// doi.org/10.1038/s41598-018-31323-7

La Shu S, Matsuzaki J, Want MY et al (2020) An immunosuppressive effect of melanoma-derived exosomes on NY-ESO-1 antigen-specific human CD8+ T cells is dependent on IL-10 and independent of BRAFV600E mutation in melanoma cell lines. Immunol Investig 49:744-757. https://doi.org/10.1080/ 08820139.2020 .1803353

Leblanc GA (2004) Basics of environmental toxicology. In: Hodgson E (ed) A textbook of modern toxicology. Wiley, New York, pp 463-478

Liebeke M, Strittmatter N, Fearn S et al (2015) Unique metabolites protect earthworms against plant polyphenols. Nat Commun 6:4-10. https://doi.org/10.1038/ncomms8869

Maltsev DI, Mellanson KA, Belousov VV, Enikolopov GN, Podgorny OV (2022) The bioavailability time of commonly used thymidine analogues after intraperitoneal delivery in mice: labeling kinetics in vivo and clearance from blood serum. Histochem Cell Biol. https://doi.org/10.1007/s00418-021-02048-y

Manders EMM, Stap J, Strackee J et al (1996) Dynamic behavior of DNA replication domains. Exp Cell Res 226:328-335. https://doi. org/10.1006/excr.1996.0233

Mayilswami S, Krishnan K, Naidu R, Megharaj M (2017) Transcriptome analysis of Eisenia fetida chronically exposed to benzo(a) pyrene. Environ Technol Innov 7:54-62. https://doi.org/10.1016/j. eti.2016.12.002

Pan BT, Johnstone RM (1983) Fate of the transferrin receptor during maturation of sheep reticulocytes in vitro: selective externalization of the receptor. Cell 33:967-978. https://doi.org/10.1016/ 0092-8674(83)90040-5

Podgorny O, Peunova N, Park J-H, Enikolopov G (2018) Triple S-phase labeling of dividing stem cells. Stem Cell Rep 10:615626. https://doi.org/10.1016/j.stemcr.2017.12.020

Rak J (2010) Microparticles in cancer. Semin Thromb Hemost 36:888906. https://doi.org/10.1055/s-0030-1267043

Raposo G, Stoorvogel W (2013) Extracellular vesicles: exosomes, microvesicles, and friends. J Cell Biol 200:373-383. https://doi. org/10.1083/jcb.201211138

Ritschar S, Schirmer E, Hufnagl B et al (2022) Classification of target tissues of Eisenia fetida using sequential multimodal chemical analysis and machine learning. Histochem Cell Biol. https://doi. org/10.1007/s00418-021-02037-1

Sparvero LJ, Amoscato AA, Dixon CE et al (2012) Mapping of phospholipids by MALDI imaging (MALDI-MSI): realities and expectations. Chem Phys Lipids 165:545-562. https://doi.org/10.1016/j. chemphyslip.2012.06.001

Strnadová K, Pfeiferová L, Přikryl P et al (2022) Exosomes produced by melanoma cells significantly influence the biological properties of normal and cancer-associated fibroblasts. Histochem Cell Biol. https://doi.org/10.1007/s00418-021-02052-2

Yao L, Li K, Peng W et al (2014) An aberrant spliced transcript of focal adhesion kinase is exclusively expressed in human breast cancer. J Transl Med 12:136. https://doi.org/10.1186/1479-5876-12-136

Zhou J, Yi Q, Tang L (2019) The roles of nuclear focal adhesion kinase (FAK) on cancer: a focused review. J Exp Clin Cancer Res 38:250. https://doi.org/10.1186/s13046-019-1265-1

Publisher's Note Springer Nature remains neutral with regard to jurisdictional claims in published maps and institutional affiliations. 